Cahiers Charlevoix

Études franco-ontariennes
Cahiers Charlevoix Études franco-ontariennes

or Cherloceris of"

\title{
Fidélité à soi et au devenir franco-ontarien : Fernand Dorais essayiste
}

\section{Michel Gaulin}

Volume 3, 1998

URI : https://id.erudit.org/iderudit/1039395ar

DOI : https://doi.org/10.7202/1039395ar

Aller au sommaire du numéro

\section{Éditeur(s)}

Société Charlevoix

Presses de l’Université d’Ottawa

ISSN

1203-4371 (imprimé)

2371-6878 (numérique)

Découvrir la revue

Citer cet article

Gaulin, M. (1998). Fidélité à soi et au devenir franco-ontarien : Fernand Dorais essayiste. Cahiers Charlevoix, 3, 179-196. https://doi.org/10.7202/1039395ar
Résumé de l'article

Michel Gaulin propose de «faire le point sur l'essentiel de la pensée » de Fernand Dorais, telle qu'il l'a pu dégager des deux recueils d'essais publiés par ce dernier : Entre Montréal... et Sudbury et Témoins d'errances en Ontario français. Il met en perspective l'activité de ce professeur de littérature de l'Université Laurentienne et la réflexion que l'essayiste entretint sur son métier de pédagogue et sur la matière qui était sienne. Il aborde ainsi la conception de la littérature de ce " penseur provocant " et l'idée qu'il se faisait $\mathrm{du}$ " devenir franco-ontarien ». Cet article est un hommage que Michel Gaulin rend à l'actualité des idées de cet auteur. 


\section{Fidélité À SOI ET AU DEVENIR FRANCO-ONTARIEN: \\ FERNAND DORAIS ESSAYISTE}

Michel Gaulin

Département d'études françaises

et College of the Humanities

Université Carleton, Ottawa

Cahiers Charlevoix 3, 1998, pp. 179-196. 


\section{SOMMAIRE}

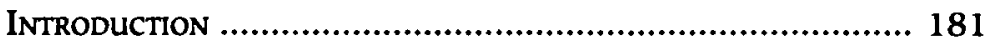

I - LA LITTÉRATURE COMME MAITRESSE DE VIE

ET D'EXPÉRIENCE ................................................... 186

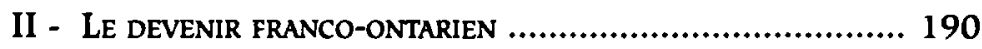

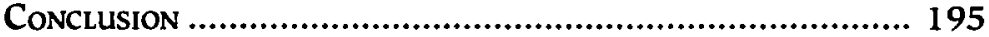




\title{
FIDÉLITÉ À SOI ET AU DEVENIR FRANCO-ONTARIEN: FERNAND Dorais esSAYISTE*
}

\author{
Si nous ne naissons pas, du moins pas encore, \\ c'est nous qui manquons d'imagination, trop peureux \\ et encore trop graine d'esclave, pour nous ouvrir inconditionnelle- \\ ment au Devenir polyvalent, toujours inouï et inédit. \\ F.D., «Représenter les francophones en \\ cette province - de la culture au cul-de-sac's, \\ Entre Montréal... et Sudbury. Pré-textes pour une \\ francophonie ontarienne, p. 91.
}

\section{INTRODUCTION}

L'odyssée intellectuelle et, pourrait-on dire, affective de Fernand Dorais, à partir du mitan de la vie, est celle d'un homme qui a pris fait et cause pour la francophonie ontarienne et son devenir historique. Arrivé à Sudbury à l'âge de quarante et un ans, en 1969, Dorais devait y rester plus d'un quart de siècle et y faire le gros de sa carrière, en tant que professeur de littérature à l'université Laurentienne. Jésuite, né

- Je tiens à remercier mon ami M.A., dont les conversations m'ont aidé à insuffler forme à ce texte. 
et formé au Québec, il figure donc parmi les derniers de cette longue lignée de ses sujets que, dans ses insondables desseins, la Compagnie envoyait depuis les débuts du siècle, soit depuis la fondation du collège du Sacré-Coeur en 1913, dans cette petite ville âpre du nord de l'Ontario pour y poursuivre, auprès de la jeunesse, son œuvre d'éducation secondaire et postsecondaire. Plusieurs ne devaient qu'y passer en route souvent vers autre chose - (Hertel en demeure, dans nos lettres, l'exemple le plus célèbre), d'autres, au contraire, tel Fernand Dorais, y rester et y prendre racine.

Pour la plupart des «élus», cependant, l'obédience sudburoise faisait figure d'exil, et Dorais lui-même - à l'origine tout au moins - n'échapperait pas à cette impression. À l'instar de Hertel, quelque trente ans plus tôt, il devait avoir longtemps, lui aussi, le sentiment $\mathrm{d}^{\text {' }}$ "errer dans Césarée ${ }^{1}$ » et vingt ans après, son arrivée devait encore lui arracher ce cri du cœur: "Quelles découvertes d'horreur ne m'y attendaient pas $!^{2} »$. Pourtant, vue à la lumière du long terme et de l'expérience, cette altération du cours de sa vie ne devait pas se révéler tant comme un exil que comme l'occasion d'une relance et d'un approfondissement, celle aussi d'une focalisation de ses "pulsions anarchiques $^{3}$ " sur l'avenir de la communauté francoontarienne.

Au moment où Dorais arrive à Sudbury, l'Ontario français est à un tournant décisif de son évolution. La rupture - idéologique autant qu'affective - avec

\footnotetext{
'Fernand Dorais, «Une expérience comme introduction", Témoins d'errances en Ontario français, p. 18. L'expression est de Racine dans Bérénice.

2 Ibid., p. 17.

${ }^{3}$ Ibid., p. 18.
} 
le Québec est à toutes fins utiles consommée, la province voisine ayant entre-temps vécu sa révolution tranquille et choisi de poursuivre dorénavant son chemin dans une direction de plus en plus divergente du reste de la francophonie canadienne. Longtemps à la remorque du Québec, tout au moins sur le plan culturel, la communauté franco-ontarienne est alors confrontée à la dure nécessité de redéfinir son identité, tâche d'autant plus difficile que cette société encore profondément conservatrice aura à composer, au surplus, avec le phénomène de la contre-culture qui secoue, à la même époque, le monde occidental. C'est dans ce contexte que, telle celle d'un prophète criant dans le désert, la voix de Fernand Dorais se fera entendre, appelant les FrancoOntariens au courage - et à l'aventure - de leur devenir collectif. La littérature, et les leçons qui s'en dégagent, seront au cour du message qu'il n'aura de cesse de livrer.

Issu du cours classique tel qu'il existait encore au Québec dans les années quarante, donc doté au départ d'une solide culture générale qu'il se chargera lui-même d'étendre et d'approfondir par de vastes lectures, Fernand Dorais s'est en effet rapidement mis à l'écoute de la littérature comme maîtresse de vie et d'expérience. C'est à son contact principalement que se forgera sa morale, faite tout à la fois de fidélité - à soi au premier chef - et d'un pragmatisme de bon aloi qui, de préférence à la théorie désincarnée, l'amènera à privilégier, comme champ d'action, le hic et nunc de son milieu de vie.

Universitaire, mais en milieu minoritaire et éloigné des grands centres de la vie intellectuelle, Dorais n'a pas tant eu l'ambition de se constituer grand érudit, 
que celle, plus modeste, et peut-être plus riche aussi sur le plan simplement humain, de laisser sa marque en tant que pédagogue - dans le sens le plus ancien et le plus noble du terme. Préoccupé avant tout du bien de ses étudiants et du prolongement dans leur vie, comme dans leur communauté d'appartenance, de l'enseignement qui leur était dispensé, Dorais s'est livré, tout au long de sa carrière, à une réflexion intense sur son métier de professeur et sur la matière qui faisait l'objet premier de son enseignement, la littérature. Au gré des circonstances et des invitations - colloques, manifestations culturelles de divers ordres, controverses - , il s'est prêté également à des formes d'intervention davantage ponctuelles et publiques qui lui ont permis de faire le point de ses observations sur la communauté franco-ontarienne, de hasarder un diagnostic, de suggérer, à l'occasion, des remèdes.

Une forme littéraire, celle de l'essai (à laquelle il s'est d'ailleurs intéressé professionnellement), semblait particulièrement indiquée pour véhiculer son propos. Par la liberté qu'elle suppose en effet, cette forme convenait parfaitement à la pensée de Dorais, pensée questionneuse faite tout à la fois de poussées en avant et de retours sur soi, pensée fidèle à sa propre subjectivité et aux dictées de son désir, mais pensée décapante aussi, qui n'a pas peur d'aller audelà des apparences pour remettre en question le statu quo. Aussi le gros de son œuvre écrite a-t-il été recueilli en deux volumes d'essais, Entre Montréal... et Sudbury. Pré-textes pour une francophonie ontarienne, paru en $1984 \mathrm{chez}$ Prise de parole 4 , et Témoins d'errances en Ontario français. Réflexions

\footnotetext{
${ }^{4}$ Fernand Dorais, Entre Montréal... et Sudbury. Pré-textes pour une francophonie ontarienne, Sudbury, Prise de parole, 1984, $168 \mathrm{p}$.
} 
venues de l'amer, publié aux Éditions du Nordir en $1990^{5}$.

Forme dans et par laquelle le "je» se constitue en sujet, l'essai permettait en outre à Dorais de faire le lien entre l'individuel et le collectif - car le "je" peut aussi, à l'occasion, être un "nous" - , de tirer de sa propre expérience des leçons instructives pour l'avenir de la collectivité franco-ontarienne, elle aussi confrontée, dans le contexte de la nouvelle donne de cette fin de siècle, à la tâche de se constituer en sujet autonome. C'est ainsi que Dorais explique pourquoi il a à deux reprises accepté que soient recueillis en volume, par les soins d'amis attentionnés, les textes disparates donnés ici et là au cours des années: "établir une continuité dans la fidélité, en prenant pour point d'appui un "cas vécu" quelconque qui pourrait, indépendamment du pauvre petit individu exhibé sans intérêt, servir de support à une réflexion à vocation exemplaire ${ }^{\star}$. Ainsi donc, c'est par la littérature, et grâce à une forme littéraire particulièrement souple qui autorise toutes les explorations, l'essai, que Fernand Dorais aura d'abord vu clair en lui-même, qu'il sera ensuite entré en rapport avec le monde, puis avec le milieu dans lequel il était appelé à vivre. La conception qu'il se fait de la littérature sert de forme structurante à l'édifice de sa pensée.

La formule propre au recueil de textes disparates, avec les redites inévitables et les recoupements qu'elle entraîne, nous incite à opter pour un plan synthétique en deux parties, qui nous permettra néanmoins, nous l'espérons, de faire le point sur l'essentiel de la pensée

${ }^{5}$ Fernand Dorais, Témoins d'errances en Ontario français. Réflexions venues de l'amer, Hearst, Le Nordir, 1990, 152 p.

${ }^{6}$ Ibid., p. 26. 
de Dorais. Ainsi, nous aborderons en premier lieu la conception qu'il se fait de la littérature, pour en voir ensuite les prolongements dans l'idée qu'il se fait du devenir franco-ontarien.

\section{I - LA LITTERATURE COMME MAITRESSE DE VIE ET D'EXPÉRIENCE}

Dorais s'est expliqué principalement sur sa conception de la littérature dans un essai intitulé «Qu'est-ce qu'on fait quand on fait de la littérature?», qui constitue l'une des pièces maîtresses de Témoins d'errances... ${ }^{7}$. Cette conception s'inspire dans une large mesure de la pensée de Freud et de la distinction que le fondateur de la psychanalyse établissait entre le principe de réalité et le principe de plaisir (ce que Jung, de son côté, désignera sous les vocables d'animus et d'anima). Assujetti, pour les besoins de la vie en société, aux impératifs du principe de réalité, l'être humain n'en a pas moins besoin, pour mener une existence équilibrée, de vivre également en harmonie avec l'autre part de lui-même, celle du "monde du désir, innombrable, innommable, pluriel, polyvalent, contradictoire ${ }^{8} "$.

C'est de cette seconde instance que relève la littérature en ce qu'elle donne libre cours à l'affectivité («les affects, les pulsions, les décharges d'énergie, qui jouent le rôle de moteur dans la création des ouvres ${ }^{9} »$ ), dont l'imaginaire se chargera par la suite de transformer les manifestations en "représentations exemplaires ${ }^{10}$ ",

7 "Qu'est-ce qu'on fait quand on fait de la littérature?», Témoins d'errances..., p. 31-61.

Ibid., p. 35.

'Loc. cit.

${ }^{10}$ Ibid., p. 36. 
auxquelles, dans un troisième temps, le langage conférera une forme.

Dorais tourne ensuite son attention du côté du lecteur (ou du spectateur, s'il s'agit, par exemple, du théâtre) pour expliquer, par le moyen des concepts aristotéliciens de la mimésis et de la catharsis, comment, au contact de l'œuvre d'art,

il [le lecteur ou le spectateur] apprivoise, pour ainsi dire, tout le monde des affects en lui: en le nommant, en le revivant, en le prenant à son propre compte et en charge, en l'explorant ainsi à fond, en n'en ayant pas peur, et enfin et surtout, par là, en l'humanisant, désamorçant ainsi l'angoisse que comportent toujours et l'acte d'exister et le fait de se trouver devant l'inconnu menaçant au fond de son être ${ }^{11}$.

Ainsi donc, la rencontre avec l'œuvre littéraire (ou, de façon plus large, avec toute œuvre d'art) entraîne, de la part de chaque individu, une réponse éminemment personnelle qui, se situant au niveau de l'expérience, lui «permet de devenir ou de demeurer humain ${ }^{12} »$.

Mais les affects n'ont jamais guère eu bonne réputation auprès de la société, qui en a toujours craint les manifestations intempestives et les débordements. De tout temps, en effet, les affects ont eu sans cesse à se défendre contre la volonté de censure ${ }^{13}$ qui fut le fait aussi bien de l'État que de l'Église. Sur cette question, Dorais prend une position ferme, qui consiste à «affirmer et à défendre la liberté inconditionnelle du

\footnotetext{
"I Ibid., p. 43.

${ }^{12}$ Loc. cit.

${ }^{13}$ Sur cette importante question, on se reportera également à l'essai intitule «La "farce" des Rogers. L'imaginaire et sa censure", dans Témoins d'errances..., p. 109-132, où Dorais aborde aussi le problème de la pornographie.
} 
créateur ${ }^{14} » . S^{\prime}$ il refuse par après de s'engager dans le débat qui consiste à savoir "comment telle ou telle œuvre d'art peut être insérée dans le tissu social de telle communauté ${ }^{15}$ ", avouant candidement ne pas avoir de solution à proposer à ce problème, il porte quant à lui la question sur un autre plan, beaucoup plus important à ses yeux, celui de la distinction entre la morale et l'éthique. Alors qu'il voit la morale comme un phénomène essentiellement externe, Dorais envisage l'éthique, elle, comme relevant du for interne. L'éthique est en effet, à ses yeux:

la loi intérieure selon laquelle par essais et erreurs l'Homme part à la recherche d'un sens, se livre à une éducation de son moi intime, découvre et construit son affectivité, exprime et éduque son imaginaire, trouve ses convictions et ses valeurs à lui ${ }^{16}$.

Le défi que lance pareille conception de l'éthique sera donc celui d'un "beau risque» et c'est celui, selon Dorais, auquel, depuis les temps les plus anciens, l'Occident aurait répondu en choisissant d'éduquer les générations successives de ses jeunesses «en leur mettant d'abord et avant tout des textes littéraires "beaux" entre les mains ${ }^{17}$ ». "L'Occident, poursuit-il par ailleurs, n'a pas pensé d'abord exclusion, censure, morale; il a d'abord pensé formation humaine par exploration de l'Humain $[\ldots]^{18}{ }_{n}$, attitude à laquelle Dorais souscrit sans réserve et dont il sent le besoin de rappeler la pertinence à une société qui, à peine sortie de son conservatisme d'antan, est fortement tentée par les appels du néoconservatisme

\footnotetext{
14 "Qu'est-ce qu'on fait quand on fait de la littérature?", Témoins d'errances..., p. 52. C'est Dorais qui souligne.

${ }^{15}$ Loc. cit.

${ }^{16}$ Loc. cit.

${ }^{17}$ Ibid., p. 44.

${ }^{18}$ Loc. cit. 
moral et une conception davantage utilitaire et égalitariste de l'éducation.

Car la tentation de censurer n'est plus en effet le seul danger qui menace la séculaire conception humaniste de la formation de la jeunesse. Aussi Dorais est-il amené à dénoncer la tendance croissante à favoriser, dans l'enseignement universitaire, «la seule information technologique ${ }^{19}$ " aux dépens des disciplines fondamentales (notamment par la place de plus en plus grande faite aux facultés dites «professionnelles"). Il s'en prend également au faux égalitarisme qui dénonce a priori toute notion de culture comme élitiste, qui, en littérature, prône l'égalité des genres (la bande dessinée contre la tragédie grecque; la chanson, pourrait-on ajouter, contre la poésie...) ou, en anthropologie, l'égalité absolue des cultures. À ce propos, Dorais rappelle opportunément que toute éducation bien comprise a toujours, en réalité, été élitiste parce que son objet est la qualité, celle de l'expérience humaine prise dans son ensemble. "L'éducation, affirme-t-il, doit demeurer l'initiation à la qualité du Langage, de l'Être humain, de la $\mathrm{Vie}^{20}$ » et même dans l'univers éclaté de cette fin de siècle, les arts et les lettres continuent à lui paraître aptes à garantir cette qualité, même s'il leur faut, pour ce faire, encourir l'odieux d'une accusation d'élitisme.

Cela étant dit, toutefois, Dorais n'en prône pas pour autant une éducation humaniste désincarnée par rapport au milieu dans lequel elle s'exerce. Dès lors que la littérature est envisagée comme uun art, un style et une forme de pensée et de vie ${ }^{21} »$, il lui

\footnotetext{
${ }^{19}$ Ibid., p. 48.

20 Ibid., p. 50.

${ }^{21}$ Ibid., p. 48. C'est Dorais qui souligne.
} 
incombe de s'adapter au milieu et aux besoins de ceux qui désirent se mettre à son école. C'est pourquoi Dorais dénoncera comme "terrorisme intellectuel $^{22}$ » la manie des "méthodes» qui s'est emparée de l'enseignement de la littérature au tournant des années 1960, parce que, à son avis, axées comme elles le sont sur le fonctionnement du texte plutôt que sur le plaisir qu'il est apte à procurer, les méthodes détournent la littérature de son caractère d'expérience humaine propre à faire " découvrir des choses encore plus secrètes dans l'homme ${ }^{23}$ ». De même, il prônera l'urgence de concevoir dans les milieux mêmes auxquels ils sont destinés les manuels devant servir à l'enseignement des lettres, manuels qui, tout en tenant compte des développements de la recherche au niveau international, chercheraient, par exemple, s'agissant de l'Ontario français, à «se réapproprier, à notre mesure, en un langage qui soit nôtre, la littérature française, pour mieux la faire comprendre à nos étudiants ${ }^{24}$ ».

C'est ce même pragmatisme empreint de réflexion qui caractérisera la pensée de Fernand Dorais en ce qui a trait à l'avenir de la collectivité francoontarienne.

\section{II - LE DEVENIR FRANCO-ONTARIEN}

L'expérience ontarienne de Fernand Dorais ne fut pas celle d'un professeur qui se serait muré dans sa discipline et qui serait resté indifférent à ce qui se passait autour de lui. Elle fut aussi, comme il l'affirme aux première pages de Témoins d'errances...,

\footnotetext{
${ }^{22}$ Ibid., p. 44.

${ }^{23}$ Loc. cit.

${ }^{24}$ Ibid., p. 46-47.
} 
"politique ${ }^{25}$ ". Dorais a été, au cours de sa carrière, mêlé de près aux diverses controverses qui ont marqué la difficile évolution de l'université Laurentienne. À l'extérieur de la communauté universitaire proprement dite, il a suivi avec attention - souvent en y prenant une part active - toutes les manifestations de dynamisme par lesquelles la communauté élargie a tenté, suite à la coupure affective d'avec le Québec, de prendre en main la maîtrise de son propre destin. Il convient toutefois de préciser d'emblée que l'expérience ontaroise de Dorais se limite surtout au nord de la province et que les observations qui s'en dégagent ne sauraient être nécessairement valables pour l'ensemble de la communauté, qui fait dorénavant figure de communauté de plus en plus éclatée en fonction de ses trois axes principaux, Sudbury, Ottawa et Toronto, cela sans parler de regroupements moins importants localisés dans d'autres régions de la province, mais ayant tout de même aussi leur personnalité propre.

Sur un plan personnel, l'expérience ontaroise de Dorais devait se solder par un retour au nationalisme de sa jeunesse, nationalisme qu'il avait pourtant, à une certaine époque, fini par honnir. Jeune religieux, en effet, Dorais, avec bien des hommes de sa génération, avait, au nom de l'appel de la vie (plutôt, sans doute, que celui de la "race»), et dans la mouvance du renouveau que représentait, par exemple, la revue Cité libre, rejeté ce qu'il appelle le "terroirisme-nationalisme ${ }^{26}$ " représenté par l'abbé Groulx et ses épigones. Or il allait, de par la situation qu'il serait appelé à vivre à la Laurentienne, être forcé de revenir, sinon à la vieille version dépassée du nationalisme, tout au moins à une variante moderne

25 «Une expérience comme introduction», Témoins d'errances, p. 23. ${ }^{26}$ Ibid., p. 15. 
qui se veut affirmation légitime de l'être profond que l'on porte en soi. Dorais affirme sans ambages, dans Témoins d'errances..., avoir été "profondément [...] écœurée ${ }^{27}$ " par l'expérience du «bilinguisme intégré» tel qu'il fut progressivement institutionnalisé à l'université et qui devait se solder, au nom de la gratuité désintéressée du savoir et d'un vague idéal d'excellence scientifique, par la prédominance de fait des intérêts de l'un des deux groupes linguistiques en présence sur ceux de l'autre. Dorais se range donc du côté de ceux, nombreux au sein de la communauté francophone du nord de la province, qui estiment que, dans l'affaire de la question universitaire dans leur région, affaire qui devait faire couler tellement d'encre au début des années soixante, les FrancoOntariens ont été entraînés dans ce qui devait s'avérer, à la longue, un marché de dupes. Au milieu des années quatre-vingt, tout au moins, Dorais croyait encore que les Franco-Ontariens «avaient un droit imprescriptible à une université française ${ }^{28}$ ", question qui est toujours, comme on sait, d'actualité.

On pourra ou non être d'accord avec l'évolution personnelle de Dorais en ce domaine qui est devenu, pour beaucoup de Canadiens français, un champ truffé de mines. C'est pourquoi nous ne nous y attarderons pas, sinon pour noter qu'il convenait cependant d'en faire état ici dans le cadre du premier des deux filons suivis dans la présente étude, celui de la fidélité à soi, principe si important dans la pensée de Fernand Dorais. Tournons donc plutôt maintenant notre atten-

\footnotetext{
$\overline{27}$ Ibid., p. 19. On pourra, pour une explication plus détaillée de la position de Dorais face à cette question, se reporter à son essai intitulé «Bilinguisme d'ici. Pratique du bilinguisme à l'Université Laurentienne», Entre Montréal... et Sudbury, p. 33-50.

28 «Bilinguisme d'ici...», Entre Montréal... et Sudbury, p. 37.
} 
tion vers le diagnostic qu'il a été amené à porter, à la lumière de ses observations, sur la francophonie ontarienne pendant les années où il en a partagé la vie.

Quand, à la fin des années soixante, la communauté franco-ontarienne est confrontée un peu malgré elle à la nécessité du changement, elle hésite entre deux voies: la récupération par ses élites - la bourgeoisie clérico-nationaliste - ou l'appel de l'aventure. La première de ces deux voies est assurément la plus réconfortante. C'est celle qui, depuis des générations, malgré des ratés épisodiques, a le mieux réussi à la communauté. Mais ces élites sont elles-mêmes déboussolées par le vent de changement qui souffle et qui, elles le sentent bien, risque de les emporter elles aussi. Dans un monde de plus en plus éclaté, où les "communications" imposent leur présence de plus en plus envahissante véhiculant sans vergogne les valeurs de la toute nouvelle contre-culture, la jeunesse a le goût de bouger et le peuple se laisse entraîner par la perspective de la fête.

Pour Fernand Dorais, cette période, qui va de 1969 à 1976 environ, est la plus passionnante. C'est celle où, sous l'impulsion des créateurs, se dessine le rêve "d'inventer un dire collectif susceptible d'établir une culture ou relancée ou naissante ${ }^{29}$ ». Mais, comme toutes les fêtes, celle-ci ne durerait qu'un temps et céderait vite la place à la désillusion. La décennie suivante apparaît en effet à Dorais comme celle d'une "descente aux enfers ${ }^{30}$ ", marquée par une assimilation galopante au sein de la jeunesse et un recul politique

\footnotetext{
${ }^{29}$ "L'Ontario francophone avant et après $1967 »$, Témoins d'errances..., p. 69.

30 «Le Nouvel-Ontario... tel que j'imagine l'avoir vécu... de 1969 à 1989 !», Témoins d'errances..., p. 80.
} 
qu'allait sanctionner l'adoption de la Constitution de 1982 , où serait finalement liquidé le «biculturalisme" des années soixante au profit du fédéralisme dit «coopératif» et de l'idéal mensonger de la réconciliation nationale au nom du "greater Canada", que Dorais attribue à Pierre Elliott Trudeau ${ }^{31}$. Pourtant, même si son constat s'arrête en 1990, année de l'échec de l'accord du lac Meech et de la parution du second volume de ses essais, Dorais semble voir, à partir du milieu des années quatre-vingt, une société qui reprend vitalité et goût à la vie.

Quand il se penche de façon plus approfondie sur les causes des divers phénomènes qu'il a observés, Dorais en vient à la conclusion que ce qui a manqué aux Franco-Ontariens, c'est la mise au point d'une pensée politique d'ensemble qui engloberait non seulement la culture (qui fut toujours leur principal point d'appui), mais également l'économique, le social et l'ethnique, trop souvent relégués au second plan. Dès son arrivée à Sudbury, c'est tout de suite à la notion d'acculturation ${ }^{32}$ que Dorais a eu recours pour expliquer les comportements qu'il voyait étalés sous ses yeux: honte de soi et de ses origines, scission de la conscience se traduisant en fidélités conflictuelles (déchirement entre la figure écrasante du Père / du Maître et celle, accommodatrice, de la Mère), hantise de l'échec. Or, les combats de libération qui ont marqué le $\mathrm{XX}^{\mathrm{e}}$ siècle nous l'ont bien montré, c'est au niveau politique - dans le sens le plus étendu du mot - que se résout le problème de l'acculturation. Malgré l'importance qu'ils ont toujours accordée à la culture, les Franco-Ontariens se révèlent, à l'ana-

\footnotetext{
${ }^{31}$ "Jean et Roberte à l'école de la culture», Témoins d'errances..., p. 98. ${ }^{32}$ "Mais qui a tué André? L'acculturation chez les Franco-Ontariens", De Montréal... à Sudbury, p. 15-33.
} 
lyse, dénués du niveau de conscience historique qui leur donnerait le champ nécessaire à un véritable combat de libération. La culture elle-même, trop longtemps collée «à quelque obscure intuition d'esthétisme ${ }^{33}$ " a besoin d'être repensée dans le sens d'un plus grand rattachement à l'expérience.

Bref, les Franco-Ontariens - tout au moins ceux du nord-est de la province, qui sont ceux que Fernand Dorais connaît le mieux - ont besoin d'un vaste programme de recherche qui leur permettrait de repenser la culture dans ce contexte plus vaste qui serait celui de ses rapports avec l'ethnicité, le nationalisme, l'idéologie, et la notion même de minorité ${ }^{34}$, mais ils devront le faire, toujours, à l'écoute des possibilités que l'imaginaire ouvre sur le «Devenir polyvalent, toujours inouï et inédit», pour reprendre les mots de la citation que nous placions en exergue à la présente étude.

\section{Conclusion}

S'il fallait maintenant résumer en quelques mots le message qui se dégage du travail de Fernand Dorais essayiste, on pourrait le faire en ces termes: faire confiance, dans la vie privée comme dans la vie publique, au désir, aux affects et à la passion qu'ils nourrissent parce qu'ils constituent le dynamisme qui préside à la vie. C'est cette confiance qui ne s'est jamais démentie chez lui qui a permis à Dorais d'intégrer en un tout harmonieux à la fois les engagements de son sacerdoce, la loyauté portée à la

\footnotetext{
${ }_{33}$ "Jean et Roberte à l'école de la culture", Témoins d'errances..., p. 103. C'est Dorais qui souligne.

${ }^{34}$ "Minorité, autonomie, dépendance. Vues d'un littérateur», Témoins d'errance..., p. 143.
} 
discipline professionnelle qu'il avait choisie et la dévotion éprouvée à l'endroit de sa communauté ethnique d'adoption, la collectivité franco-ontarienne.

L'essai, affirmait déjà Dorais, dans le premier de ses deux recueils, est «presque toujours scandaleux et provocant ${ }^{35}$ ». Dorais lui-même, par le canal de l'essai, est un penseur provocant, des textes duquel la communauté franco-ontarienne peut encore aujourd'hui tirer un programme d'action. Il mérite toujours d'être écouté.

$\overline{35}$ «La passion et la violence du marginalisé... ou Vive la différence», De Montréal... d Sudbury, p. 60. 